\title{
MODIFIED POISSON KERNELS ON RANK ONE SYMMETRIC SPACES
}

\author{
ANNA MARIA MANTERO ${ }^{1}$
}

\begin{abstract}
An extension is obtained to the case of a real rank one noncompact symmetric space $G / K$ of the solution of the following problem on half-spaces: given an arbitrary continuous function $f(x)$ on $\mathbf{R}^{n}$, is it possible to find a function $F$ on $\mathbf{R}^{n} \times \mathbf{R}^{+}$such that $F(x, y)$ is continuous for $y>0$, harmonic for $y>0$ and such that $F(x, 0)=f(x)$ ?
\end{abstract}

1. Introduction and notations. Let $G$ be a connected noncompact semisimple Lie group with finite center and of real rank one. Let $\theta$ be a Cartan involution of the Lie algebra $g$ of $G$ corresponding to the Cartan decomposition $\mathrm{g}=k \oplus \mathfrak{p}$. Let $K$ be the corresponding maximal compact subgroup of $G$ and $G / K$ the associated symmetric space. Let $G=V A K$ be an Iwasawa decomposition of $G$, so every element $g \in G$ can be written as $g=v a_{t} k$, where $v \in V, a_{t}=\exp t H \in A$ (the element $H$ in a-the Lie algebra of $A$-is chosen in such a way that $\alpha(H)=1$ ) and $k \in K$. We also denote by $H(g)$ the logarithm of the $A$-component $a_{t}$ of $g$ in a. We can write every element of $V$ as $v=\exp X \exp Y$ with $X \in g_{-\alpha}, Y \in g_{-2 \alpha}$ where $\alpha$ and $2 \alpha$ (or $\alpha$ ) denote the positive restricted roots (or root) of $(\mathfrak{g}, \mathfrak{a})$. The (real) dimensions of the root spaces $g_{-\alpha}$ and $g_{-2 \alpha}$ are denoted, respectively, by $p$ and $q$. If $B$ is the Killing form of $g$, we put $\|Y\|^{2}=-B(Y, \theta Y)$ for $Y \in g$. We also put $2 p=(p+2 q) \alpha$. For $M$ the centralizer of $A$ in $K$, the Poisson kernel $P$ is defined on $G / K \times K / M$ (or on $G / K \times B$ for some boundary $B$ isomorphic to $K / M$; cf. Koranyi [5]) by

$$
P(g K, k M)=\exp \left(-2 \rho H\left(g^{-1} k\right)\right) .
$$

Making use of the formulas on pp. 65 and 67 of Helgason's paper [3] we can consider $P$ as a function defined on $G / K \times V$ by the following expression:

$$
P\left(a_{t} K, v_{0}\right)=\exp \left(2 \rho \log a_{t}\right) \exp \left(-2 \rho H\left(a_{-t} v_{0} a_{t}\right)\right) .
$$

Here, the Poisson kernel is always given by (1).

A $C^{\infty}$ function $f$ on $G / K$ is called harmonic if it is annihilated by all left-invariant differential operators on $G / K$ without constant term. In particular, the Poisson kernel $P$ is harmonic on $G / K$ for each fixed element of $V$. It is also known that, if $f$ is a bounded continuous function on $V$ and $F$ is its Poisson integral, namely,

Received by the editors August 8, 1978 and, in revised form, December 14, 1978. AMS (MOS) subject classifications (1970). Primary 43A85.

${ }^{1}$ The author wishes to thank N. Lohoue for suggesting this problem and for his friendship. 


$$
F(g K)=\int_{V} P\left(g K, v_{0}\right) f\left(v_{0}\right) d v_{0}
$$

where $d v_{0}$ is the appropriate Haar measure on $V$, then $F$ is harmonic on $G / K$ and $\lim _{t \rightarrow \infty} F\left(v a_{t} K\right)=f(v)$. If we think of $G / K$ as a generalization of the upper half-plane and its boundary $V$ as a generalization of the real line, this limit generalizes vertical limits in the upper half-plane. For all these definitions and notations we refer to Helgason [2].

The following theorem generalizes to rank one symmetric spaces the result obtained by Finkelstein and Scheinberg for $\mathbf{R}^{n}[1]$.

THEOREM. Let $f$ be an arbitrary continuous function on $V$. Then there exists a kernel $W_{f}$ defined on $G / K \times V$ and depending on $f$ such that:

(i) $\int_{V} W_{f}\left(v a_{t} K, v_{0}\right) f\left(v_{0}\right) d v_{0}=F\left(v a_{t} K\right)$ converges for every $v a_{t} K \in G / K$.

(ii) $\lim _{t \rightarrow \infty} F\left(v a_{t} K\right)=f(v)$, for every $v a_{t} K \in G / K$.

(iii) $F$ is a harmonic function on $G / K$.

REMARK. The theorem is still true if we replace the condition " $f$ continuous" by the condition " $f$ locally integrable".

2. The Poisson kernel. The Poisson kernel $P$ on $G / K \times V$ is in the rank one case, given by the formula (see [3, pp. 65-67])

$$
P\left(a_{t} K, v_{0}\right)=\left[\frac{e^{2 t}}{\left(1+c\left\|e^{t} X_{0}\right\|^{2}\right)^{2}+4 c\left\|e^{2 t} Y_{0}\right\|^{2}}\right]^{p / 2+q}
$$

where $v_{0}=\exp X_{0} \exp Y_{0}$ with $X_{0} \in g_{-\alpha}, Y_{0} \in g_{-2 \alpha}$ and where $c=1 / 4(p$ $+4 q$ ). If $v=\exp X \exp Y$ with $X \in \mathfrak{g}_{-\alpha}, Y \in \mathfrak{g}_{-2 \alpha}$, then

$$
v^{-1} v_{0}=\exp \left(X_{0}-X\right) \exp \left(Y_{0}-Y+\left\{X, X_{0}\right\}\right) \text {, }
$$

where $\{$,$\} denotes a bilinear form from g_{-\alpha} \times g_{-\alpha}$ into $g_{-2 \alpha}$. So, from the identity $P\left(v a_{t} K, v_{0}\right)=P\left(a_{t} K, v^{-1} v_{0}\right)$, we get

$$
P\left(v a_{t} K, v_{0}\right)=\left[\frac{e^{2 t}}{\left(1+c e^{2 t}\left\|X_{0}-X\right\|^{2}\right)^{2}+4 c e^{4 t}\left\|Y_{0}-Y+\left\{X, X_{0}\right\}\right\|^{2}}\right]^{p / 2+q}
$$

We put, for notational convenience, $\left|v_{0}\right|=c^{2}\left\|X_{0}\right\|^{4}+4 c\left\|Y_{0}\right\|^{2}$, and $\sigma=$ $-(p / 2+q)$.

Let us define

$$
Z_{1}=Z_{1}\left(v, v_{0}\right)=2 c\left[\left\|X_{0}\right\|^{2}+\|X\|^{2}+2 B\left(X_{0}, \theta X\right)\right]
$$

and

$$
\begin{gathered}
Z_{2}=Z_{2}\left(v, v_{0}\right)=c^{2}\left[\|X\|^{4}+2\left\|X_{0}\right\|^{2}\|X\|^{2}+4 B^{2}\left(X_{0}, \theta X\right)\right. \\
\left.+4\left\|X_{0}\right\|^{2} B\left(X_{0}, \theta X\right)+4\|X\|^{2} B\left(X_{0}, \theta X\right)\right] \\
+4 c\left[\|Y\|^{2}+\left\|\left\{X, X_{0}\right\}\right\|^{2}+2 B\left(Y_{0}, \theta Y\right)\right. \\
\left.-2 B\left(Y_{0}, \theta\left\{X, X_{0}\right\}\right)+2 B\left(Y, \theta\left\{X, X_{0}\right\}\right)\right]
\end{gathered}
$$


We may now write

$$
P\left(v a_{t} K, v_{0}\right)=\left(e^{2 t}\left|v_{0}\right|\right)^{\sigma}(1+Z)^{\sigma}
$$

where

$$
Z=\frac{1+e^{2 t} Z_{1}+e^{4 t} Z_{2}}{e^{4 t}\left|v_{0}\right|},
$$

is a function on $G / K \times V$. Finally, we call $\tilde{Z}$ the expression that we obtain in (2) if we take the absolute value of each term making up $Z_{1}$ and $Z_{2}$.

Let $v a_{t} K$ be a fixed element in $G / K$. Since $\tilde{Z}$ decreases to zero when $\left|v_{0}\right|$ tends to infinity, it is possible to choose a least positive constant $C_{1}$ (depending on $\left.v a_{t} K\right)$ in such a way that, for every $v_{0} \in V$ with $\left|v_{0}\right|>C_{1}$, then $\tilde{Z}<\frac{1}{2}$. Obviously, for the same $v_{0}$ 's, also the absolute value of $Z$ is less than or equal to $\frac{1}{2}$. Thus, for every $v a_{t} K$ fixed, the following identity

$$
P\left(v a_{t} K, v_{0}\right)=\left(e^{2 t}\left|v_{0}\right|\right)^{o} \sum_{j>0}(-1)^{j} b_{j} Z^{j}
$$

where

$$
b_{j}=\frac{(-\sigma+j-1) !}{(-\sigma-1) ! j !}
$$

is satisfied for all $v_{0}$ such that $\left|v_{0}\right|>C_{1}$.

Remarks. (1) Let $v \in V$ be a fixed element and let $t$ belong to the interval $[0, \infty)$. Since the constant $C_{1}$, depending on $v a_{t} K$, decreases when $t$ goes to infinity, there exists a least positive constant $C_{2}$, depending only on $v$, such that if $v_{0} \in V$ and $\left|v_{0}\right|>C_{2}$, then $\tilde{Z}<\frac{1}{2}$ for every positive $t$.

(2) If $H$ is a compact set in $G / K$, it is possible to choose a positive constant $C_{3}$, depending on $H$, in such a way that for all $v_{0} \in V$ with $\left|v_{0}\right|>C_{3}$, then $\tilde{Z}<\frac{1}{2}$ for every $v a_{t} K \in H$.

(3) Obviously the series $\Sigma_{j>0}(-1)^{j} b_{j} Z^{j}$ converges where the series $\Sigma_{j>0} b_{j} \tilde{Z}^{j}$ converges. Moreover, the series $\Sigma_{j>0} b_{j} 2^{-j}$ is convergent.

In order to reorder the series expression for the Poisson kernel $P$, we consider the action of $A$ on $V$ defined by

$$
a_{s} \cdot v_{0}{ }^{n}=\exp \left(e^{s} X_{0}\right) \exp \left(e^{2 s} Y_{0}\right)
$$

where $v_{0}=\exp X_{0} \exp Y_{0}$. We assume also $s>0$. The expression of $P$ as a function on $G / K \times(A \cdot V)$ is then the following:

$$
\begin{aligned}
P\left(v a_{t} K, a_{s} \cdot v_{0}\right) & \\
& =\left[\frac{e^{2 t}}{\left(1+c e^{2 t}\left\|e^{s} X_{0}-X\right\|^{2}\right)^{2}+4 c e^{4 t}\left\|e^{2 s} Y_{0}-Y+e^{s}\left\{X, X_{0}\right\}\right\|^{2}}\right]^{-\sigma} .
\end{aligned}
$$

Let us define

$$
R_{1}=R_{1}\left(v, a_{s} \cdot v_{0}\right)=2 c\left[e^{2 s}\left\|X_{0}\right\|^{2}+\|X\|^{2}+2 e^{s} B\left(X_{0}, \theta X\right)\right]
$$


and

$$
\begin{gathered}
R_{2}=R_{2}\left(v, a_{s} \cdot v_{0}\right)=c^{2}\left[\|X\|^{4}+2 e^{2 s}\left\|X_{0}\right\|^{2}\|X\|^{2}+4 e^{2 s} B^{2}\left(X_{0}, \theta X\right)\right. \\
\left.+4 e^{3 s}\left\|X_{0}\right\|^{2} B\left(X_{0}, \theta X\right)+4 e^{s}\|X\|^{2} B\left(X_{0}, \theta X\right)\right] \\
+4 c\left[\|Y\|^{2}+e^{2 s}\left\|\left\{X, X_{0}\right\}\right\|^{2}+2 e^{2 s} B\left(Y_{0}, \theta Y\right)\right. \\
\left.-2 e^{3 s} B\left(Y_{0}, \theta\left\{X, X_{0}\right\}\right)+2 e^{s} B\left(Y, \theta\left\{X, X_{0}\right\}\right)\right] .
\end{gathered}
$$

So we can express

$$
\begin{aligned}
P\left(v a_{t} K, a_{s} \cdot v_{0}\right) & =\left(e^{2(t+2 s)}\left|v_{0}\right|\right)^{\sigma}[1+R]^{\sigma} \\
& =\left(e^{2(t+2 s)}\left|v_{0}\right|\right)^{\sigma} \sum_{j>0}(-1)^{j} b_{j} R^{j},
\end{aligned}
$$

where

$$
R=\frac{1+e^{2 t} R_{1}+e^{4 t} R_{2}}{e^{4(t+s)}\left|v_{0}\right|},
$$

is a function on $G / K \times(A \cdot V)$. Collecting together the elements of the sum with the same homogeneity in $e^{s}$, we get

$$
\begin{aligned}
P\left(v a_{t} K, a_{s} \cdot v_{0}\right) & =\left(e^{2(t+2 s)}\left|v_{0}\right|\right)^{\sigma} \sum_{j>0} h_{j}\left(v a_{t} K, v_{0}\right) e^{-j s} \\
& =\left(e^{2 t}\left|v_{0}\right|\right)^{\sigma} \sum_{j>0} h_{j}\left(v a_{t} K, v_{0}\right) e^{(4 \sigma-j) s}
\end{aligned}
$$

where the functions $h_{j}$ are defined in such a way that the identity is true when the series is convergent. We define also

$$
H_{j}\left(v a_{t} K, v_{0}\right)=\left(e^{2 t}\left|v_{0}\right|\right)^{o} h_{j}\left(v a_{t} K, v_{0}\right) .
$$

If, in particular, $s=0$ we have

$$
P\left(v a_{t} K, v_{0}\right)=\sum_{j>0} H_{j}\left(v a_{t} K, v_{0}\right)
$$

3. The $W_{f}$ kernel. Let $H_{j}: G / K \times V \rightarrow \mathbf{R}$ be the functions defined in $\$ 2$. They are piecewise continuous and bounded on $V$. Now we want to prove that they are also harmonic as functions on $G / K$. Let $v_{0}$ be a fixed element in $V$. Then, for every $v a_{t} K$ in $G / K$ we choose a positive real number $s$ large enough so that the following identity holds:

$$
P\left(v a_{t} K, a_{s} \cdot v_{0}\right)=\sum_{j>0} H_{j}\left(v a_{t} K, v_{0}\right) e^{(4 \sigma-j) s} .
$$

Taking the limit on both sides as $s$ tends to infinity we find

$$
H_{0}\left(v a_{t} K, v_{0}\right)=\lim _{s \rightarrow \infty} P\left(v a_{t} K, a_{s} \cdot v_{0}\right) e^{4 a s} \text {. }
$$

Since for every fixed $s$ the function $P$ is harmonic, $H_{0}\left(\cdot, v_{0}\right)$ is also harmonic on $G / K$. In the same way, we obtain, for $j=1,2, \ldots$, 


$$
H_{j}\left(v a_{t} K, v_{0}\right)=\lim _{s \rightarrow \infty} e^{j s}\left[P\left(v a_{t} K, a_{s} \cdot v_{0}\right) e^{4 o s}-\sum_{r=0}^{j-1} H_{r}\left(v a_{t} K, v_{0}\right) e^{-r s}\right] .
$$

The harmonicity of the function $H_{j}\left(\cdot, v_{0}\right)$ on $G / K$ is thus proved.

Let us consider now the function $f$ we want to extend. Let $\varphi$ be a positive continuous function from $V$ into $\mathbf{R}$ such that

$$
\left|f\left(v_{0}\right)\right| \varphi\left(v_{0}\right)<\left|v_{0}\right|^{-2+\sigma / 2}
$$

for every $v_{0} \in V$. Let us define a nonnegative integer valued function $J$ on $V$ by $J\left(v_{0}\right)=J_{0}$, where $J_{0}$ is the least integer for which the following inequality

$$
\sum_{j>\left(J_{0}-1\right) / 3} b_{j} 2^{-j}<\varphi\left(v_{1}\right),
$$

holds for every $v_{1} \in V$ such that $1<\left|v_{1}\right|<\left|v_{0}\right|$ and where $J_{0}=0$ if $\left|v_{0}\right|<1$. The function $J$ is well defined and depends on the choice of $\varphi$. Moreover, if $v_{1}, v_{2} \in V$ and $\left|v_{1}\right|<\left|v_{2}\right|$, then $J\left(v_{1}\right)<J\left(v_{2}\right)$.

We define now a function $Q$ on $G / K \times V$ by

$$
Q\left(v a_{t} K, v_{0}\right)=\sum_{j<J_{0}} H_{j}\left(v a_{t} K, v_{0}\right) .
$$

Finally, we define on $G / K \times V$ the kernel $W_{f}$ associated to the function $f$ by

$$
W_{f}\left(v a_{t} K, v_{0}\right)=P\left(v a_{t} K, v_{0}\right)-Q\left(v a_{t} K, v_{0}\right) \text {. }
$$

We estimate this kernel for a fixed element $v a_{t} K$ in $G / K$. Let us suppose $C_{1}>1$. For any $v_{0} \in V$ such that $\left|v_{0}\right|>C_{1}$ we have

$$
\begin{aligned}
\left|W_{f}\left(v a_{t} K, v_{0}\right)\right| & =\left|\sum_{j>J_{0}} H_{j}\left(v a_{t} K, v_{0}\right)\right| \\
& <\left(e^{2 t}\left|v_{0}\right|\right)^{o} \sum_{j>\frac{1}{3}\left(J_{0}-1\right)} b_{j} 2^{-j}<\left(e^{2 t}\left|v_{0}\right|\right)^{o} \varphi\left(v_{0}\right) .
\end{aligned}
$$

Remarks. (1) If $v \in V$ is fixed and $a_{t}$ varies in $A$ when $t$ belongs to the interval $[0, \infty)$ we have that

$$
\left|W_{f}\left(v a_{t} K, v_{0}\right)\right|<\left(e^{2 t}\left|v_{0}\right|\right)^{o} \varphi\left(v_{0}\right)
$$

for all $v_{0} \in V$ such that $\left|v_{0}\right|>C_{2}$.

(2) Analogously, when $v a_{t} K$ varies in a compact set $H$ of $G / K$, there exists a positive constant $D$ such that, for every $v_{0} \in V$ with $\left|v_{0}\right|>C_{3}$, we have

$$
\left|W_{f}\left(v a_{t} K, v_{0}\right)\right|<D \varphi\left(v_{0}\right) .
$$

4. Proof of the theorem. Let $T \in \mathbf{R}^{+}$. We define $E(T)=\left\{v_{0} \in V:\left|v_{0}\right|<\right.$ $T\}$.

(i) Let $v a_{t} K \in G / K$ be a fixed element and suppose $T>\max \left(1, C_{1}\right)$. We can write 


$$
\begin{aligned}
F\left(v a_{t} K, v_{0}\right)= & \int_{E(T)} P\left(v a_{t} K, v_{0}\right) f\left(v_{0}\right) d v_{0}-\int_{E(T)} Q\left(v a_{t} K, v_{0}\right) f\left(v_{0}\right) d v_{0} \\
& +\int_{V E(T)} W_{f}\left(v a_{t} K, v_{0}\right) f\left(v_{0}\right) d v_{0} .
\end{aligned}
$$

The first integral is finite because $P f$ is a bounded continuous function on the compact set $E(T)$. The second integral is also finite because on $E(T), Q f$ is a finite sum of bounded and measurable functions. For the third integral we have

$$
\begin{aligned}
\int_{V \backslash E(T)}\left|W_{f}\left(v a_{t} K, v_{0}\right) f\left(v_{0}\right)\right| d v_{0} & <\int_{V E(T)}\left(e^{2 t}\left|v_{0}\right|\right)^{\sigma} \varphi\left(v_{0}\right)\left|f\left(v_{0}\right)\right| d v_{0} \\
& <\left(e^{2 t}\right)^{\sigma} \int_{V \backslash E(T)}\left|v_{0}\right|^{-2+\sigma / 2} d v_{0}=\frac{e^{2 t \sigma}}{T},
\end{aligned}
$$

which is finite. So (i) is proved.

(ii) Fix $v \in V$. Take $T>\max \left(1, C_{2}\right)$ and observe that $T>|v|$. We know that

$$
\lim _{t \rightarrow \infty} \int_{V} P\left(v a_{t} K, v_{0}\right) f\left(v_{0}\right) \chi_{E(T)}\left(v_{0}\right) d v_{0}=f(v),
$$

if $\chi_{E(T)}$ is the characteristic function of the compact set $E(T)$. We can estimate

$$
\begin{aligned}
\lim _{t \rightarrow \infty}\left|\int_{E(T)} Q\left(v a_{t} K, v_{0}\right) f\left(v_{0}\right) d v_{0}\right| & \\
& <\lim _{t \rightarrow \infty}\left(e^{2 t}\left|v_{0}\right|\right)^{o} \int_{E(T)} \sum_{j<J_{0}}\left|h_{j}\left(v a_{t} K, v_{0}\right) f\left(v_{0}\right)\right| d v_{0}=0,
\end{aligned}
$$

because $\left|h_{j} f\right|$ are bounded and measurable functions on $E(T)$ and the sum is finite on $E(T)$.

Finally, we estimate

$$
\lim _{t \rightarrow \infty} \int_{V \in E(T)}\left|W_{f}\left(v a_{t} K, v_{0}\right) f\left(v_{0}\right)\right| d v_{0}<\frac{1}{T} \lim _{t \rightarrow \infty} e^{2 t \sigma}=0 .
$$

So we have proved that $\lim _{t \rightarrow \infty} F\left(v a_{t} K\right)=f(v)$, for every $v a_{t} K \in G / K$.

(iii) Let $H$ be a fixed compact set in $G / K$. Let $T$ be any integer such that $T>\max \left(1, C_{3}\right)$. We have

$$
\lim _{T \rightarrow \infty} \int_{V E(T)}\left|W_{f}\left(v a_{t} K, v_{0}\right) f\left(v_{0}\right)\right| d v_{0}<D \lim _{T \rightarrow \infty} \frac{1}{T}=0
$$

uniformly on $H$. So, uniformly on $H$, we have

$$
\begin{aligned}
F\left(v a_{t} K\right)= & \lim _{T \rightarrow \infty} \int_{E(T)} P\left(v a_{t} K, v_{0}\right) f\left(v_{0}\right) d v_{0} \\
& -\lim _{T \rightarrow \infty} \int_{E(T)} Q\left(v a_{t} K, v_{0}\right) f\left(v_{0}\right) d v_{0} .
\end{aligned}
$$


Both functions in (3) are harmonic on $G / K$, so (iii) is proved.

REMARK. The reader may wish to compare our "explicit" construction with the more general techniques of [4].

\section{REFERENCES}

1. M. Finkelstein and S. Scheinberg, Kernels for solving problems of Dirichlet type in a half plane, Advances in Math. 18 (1975), 108-113.

2. S. Helgason, Differential geometry and symmetric spaces, Academic Press, New York, 1962.

3. A duality for symmetric space with applications to group representations, Advances in Math. 5 (1970), 1-154.

4. M. Kashiwara, A. Kowata, K. Minemura, K. Okamoto, T. Oshima and M. Tanaka, Eigenfunctions of invariant differential operators on a symmetric space, Ann. of Math. 107 (1978), 1-39.

5. A. Koranyi, Boundary behavior of Poisson integrals on symmetric spaces, Trans. Amer. Math. Soc. 140 (1969), 393-409.

IstTtuto di Matematica, Via L. B. Alberti 4, 16132 Genova, Italy 